\title{
Molecular Relaxations in Supercooled Liquid and Glassy \\ States of Amorphous Quinidine: Dielectric Spectroscopy and Density Functional Theory (DFT) Approaches
}

Benjamin Schammé $\uparrow \S$, Mélanie Mignot $\uparrow$, Nicolas Couvrat $\uparrow$, Vincent Tognetti $\ddagger$, Laurent Joubert †, Valérie Dupray $\dagger^{*}$, Laurent Delbreilh $\S *$, Éric Dargent $\S$, Gérard Coquerel $\dagger$

† Normandie Univ, Laboratoire SMS - EA3233, Univ Rouen, F-76821, Mont Saint Aignan, France $\S$ AMME-LECAP EA 4528 International Lab, Av. de l'Université, BP12, Normandie Univ, Université de Rouen Normandie, 76801 St Etienne du Rouvray, France.

$\ddagger$ COBRA UMR 6014 and FR 3038, Normandie Univ, Université de Rouen, INSA Rouen, CNRS, F-76821 Mont Saint Aignan, Cedex, France.

*Corresponding authors: (+332323990 82),valerie.dupray@univ-rouen.fr

(+332329550 84),laurent.delbreilh@univ-rouen.fr

\section{Supporting Information Description}

Experimental and calculated XRPD patterns of crystalline Quinidine, thermogravimetric analysis of the as-received crystalline Quinidine, DSC thermograms of amorphous Quinidine measured immediately after preparation and after 16 months of storage at $20{ }^{\circ} \mathrm{C}\left(\mathrm{T}_{\mathrm{g}}-40{ }^{\circ} \mathrm{C}\right)$, dielectric strength $\Delta \varepsilon$ parameter of the Havriliak-Negami relaxation function as a function of temperature, symmetric and asymmetric broadening parameters of the Havriliak-Negami relaxation function as a function of temperature, visualization of energy, dipole changes and conformational interconversions before and after the sharp decrease of the dipole moment during the rotation of the $\mathrm{CH}(\mathrm{OH}) \mathrm{C}_{9} \mathrm{H}_{14} \mathrm{~N}$ group and finally the energy $(\Delta \mathrm{E})$, Gibbs free energy $(\Delta \mathrm{G})$ and dipole moment (D) for the three obtained minima and transition states with and without solvent at the $\omega \mathrm{B} 97 \mathrm{XD} / 6$ $311++\mathrm{G}(2 \mathrm{~d}, 2 \mathrm{p})$ level. Detailed description of the Broadband Dielectric Spectroscopy (BDS) experimental protocols and data fitting and QTAIM decomposition of molecular dipole moments is also provided. 


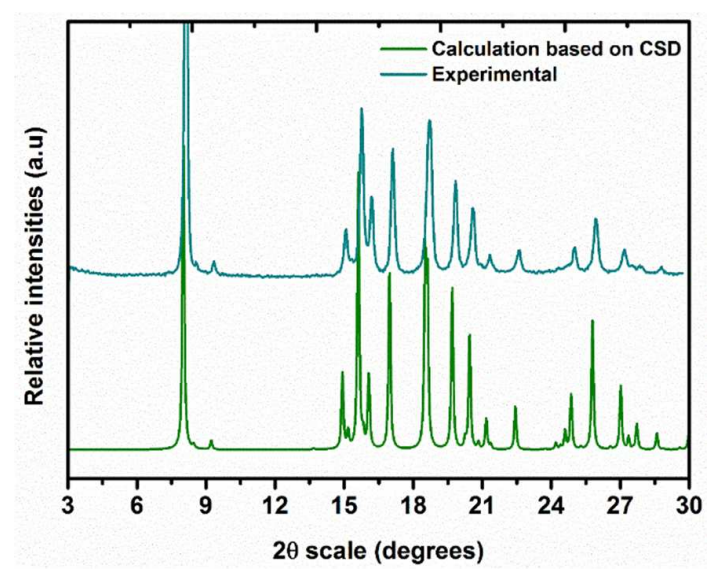

Figure S1. Experimental (blue) and calculated (green) XRPD patterns of crystalline Quinidine. Calculated pattern corresponds to the crystalline structure of Quinidine from the Cambridge Structural Database (CSD-BOMDUC).

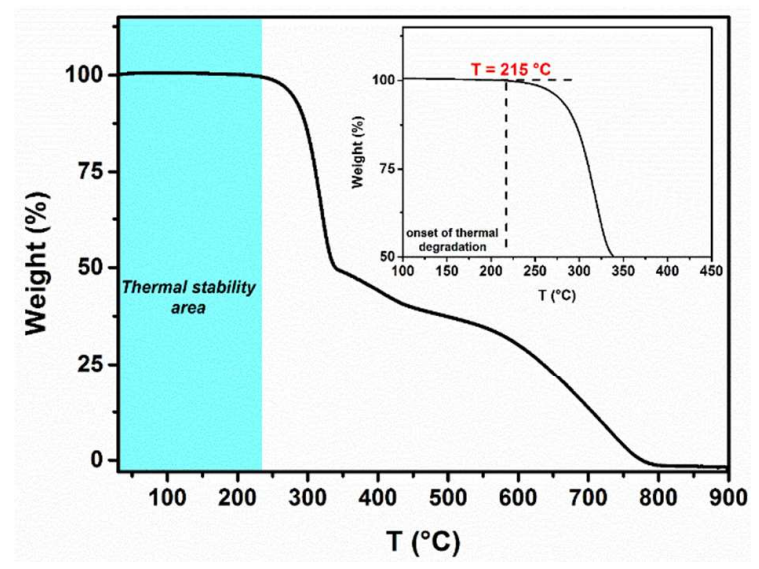

Figure S2. Thermogravimetric curve obtained on heating at $10 \mathrm{~K} / \mathrm{min}$ of the as-received crystalline Quinidine. The inset shows the onset of thermal degradation region on an enlarged scale.

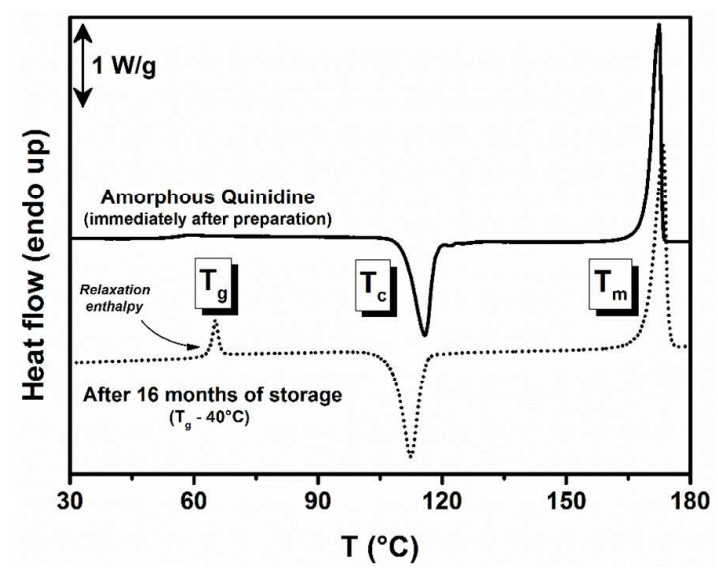


Figure S3. DSC thermograms of amorphous Quinidine measured immediately after preparation (solid line) and after 16 months of storage at $20^{\circ} \mathrm{C}\left(\mathrm{T}_{\mathrm{g}}-40^{\circ} \mathrm{C}\right)$ (short dotted line). The heating rate was of $5 \mathrm{~K} / \mathrm{min}$.

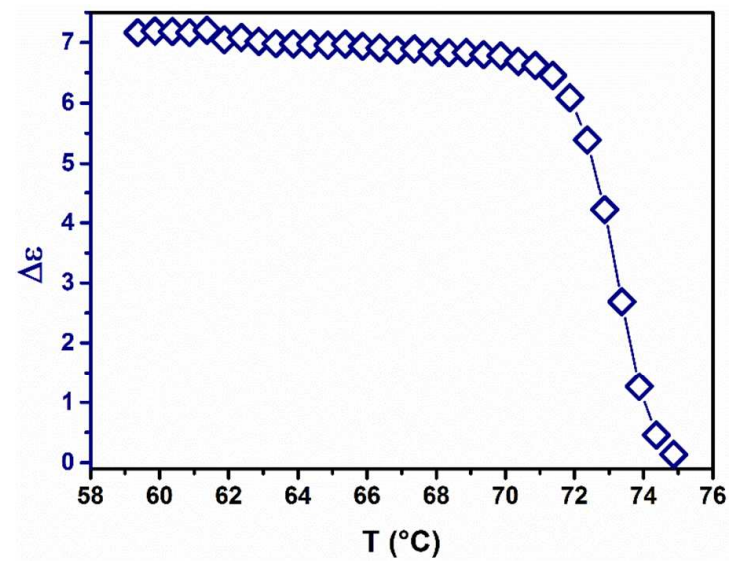

Figure S4. Dielectric strength $\Delta \varepsilon$ parameter of the Havriliak-Negami relaxation function as a function of temperature on the range of analysis.

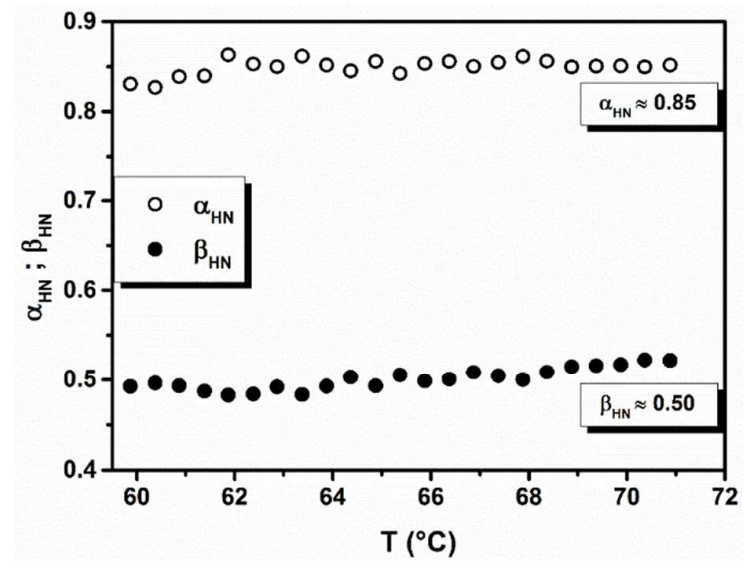

Figure S5. Symmetric $\alpha_{\mathrm{HN}}$ (open circles) and asymmetric $\beta_{\mathrm{HN}}$ (filled circles) broadening parameters of the Havriliak-Negami relaxation function as a function of temperature. 


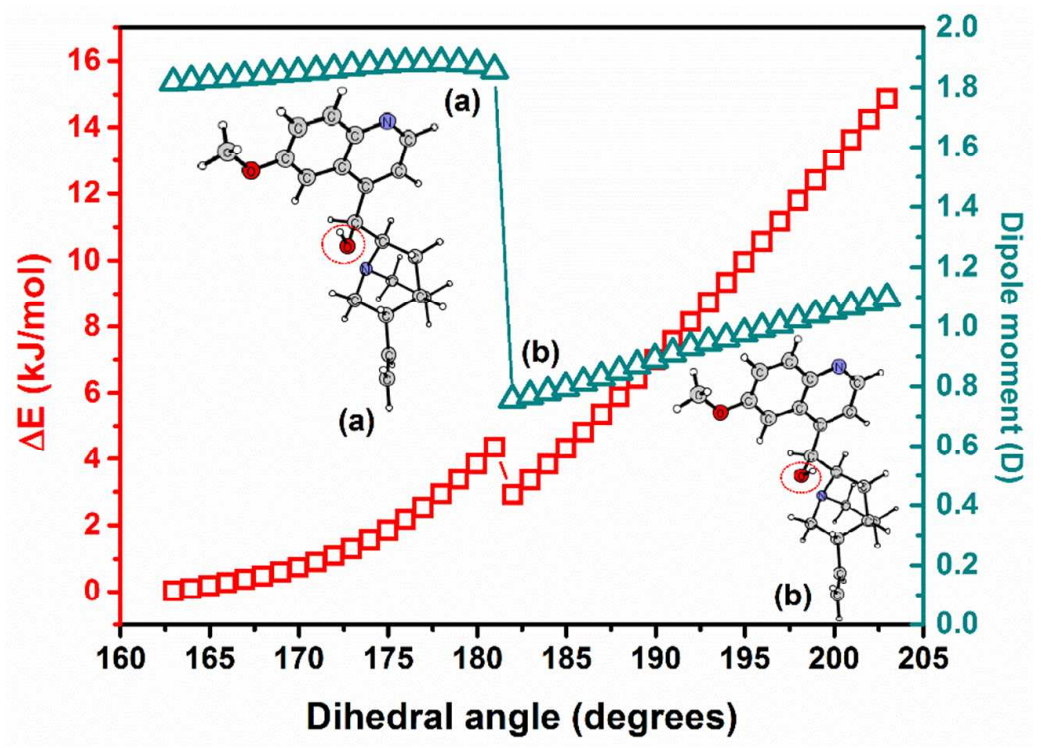

Figure S6. Visualization of energy, dipole changes and conformational interconversions (a) before and (b) after the sharp decrease of the dipole moment during the rotation of the $\mathrm{CH}(\mathrm{OH}) \mathrm{C}_{9} \mathrm{H}_{14} \mathrm{~N}$ group.

\begin{tabular}{ccccc}
$\begin{array}{c}\text { Minima } \\
\text { (MIN) }\end{array}$ & Solvent & $\begin{array}{c}\mathbf{\Delta E} \\
(\mathbf{k J} / \mathbf{m o l})\end{array}$ & $\begin{array}{c}\boldsymbol{\Delta} \mathbf{G} \\
\mathbf{( k J / m o l})\end{array}$ & $\begin{array}{c}\text { Dipole } \\
\text { moment (D) }\end{array}$ \\
& Without & 12.1 & 14.1 & 3.69 \\
\multirow{3}{*}{ MIN1 } & Heptane & 9.9 & 11.2 & 4.10 \\
& Methanol & 3.7 & 4.3 & 4.86 \\
& Water & 3.0 & 3.7 & 4.90 \\
& Without & 0.0 & 0.0 & 1.86 \\
MIN2 & Heptane & 0.0 & 0.0 & 2.09 \\
& Methanol & 0.0 & 0.0 & 2.42 \\
& Water & 0.0 & 0.0 & 2.43 \\
& Without & 13.7 & 14.8 & 1.72 \\
MIN3 & Heptane & 14.3 & 16.0 & 1.84 \\
& Methanol & 14.8 & 13.7 & 1.97 \\
& Water & 14.8 & 13.4 & 1.98
\end{tabular}

\begin{tabular}{ccccc|}
$\begin{array}{c}\text { Transition } \\
\text { states (TS) }\end{array}$ & Solvent & $\begin{array}{c}\Delta \mathbf{E} \\
(\mathbf{k J} / \mathbf{m o l})\end{array}$ & $\begin{array}{c}\Delta \mathbf{G} \\
(\mathbf{k J} / \mathbf{m o l})\end{array}$ & $\begin{array}{c}\text { Dipole } \\
\text { moment (D) }\end{array}$ \\
& Without & 50.2 & 57.4 & 4.46 \\
\multirow{2}{*}{ TS1 } & Heptane & 48.7 & 55.3 & 4.94 \\
& Methanol & 44.9 & 49.7 & 5.52 \\
& Water & 44.7 & 49.0 & 5.54 \\
TS2 & Without & 17.4 & 21.7 & 1.70 \\
& Heptane & 17.5 & 21.6 & 1.87
\end{tabular}




$\begin{array}{ccccc} & \text { Methanol } & 17.2 & 19.3 & 2.17 \\ & \text { Water } & 17.1 & 19.2 & 2.19 \\ & \text { Without } & 36.7 & 44.3 & 1.75 \\ & \text { Heptane } & 36.3 & 42.2 & 1.94 \\ \text { TS3 } & \text { Methanol } & 33.9 & 39.1 & 2.44 \\ & \text { Water } & 33.8 & 38.9 & 2.48\end{array}$

Table S1. Energy $(\Delta \mathrm{E})$, Gibbs free energy $(\Delta \mathrm{G})$ and dipole moment (D) for the three obtained minima and transition states with and without solvent at the $\omega \mathrm{B} 97 \mathrm{XD} / 6-311++\mathrm{G}(2 \mathrm{~d}, 2 \mathrm{p})$ level.

\section{Detailed description of the Broadband Dielectric Spectroscopy (BDS) experimental protocols} and data fitting:

Dielectric relaxation spectra were analyzed by means of the empirical Havriliak-Negami (HN) function with a dc-conductivity term added if necessary to fit more precisely experimental data:

$$
\varepsilon_{H N}^{*}(\omega)=\varepsilon^{\prime}(\omega)-i \varepsilon^{\prime \prime}(\omega)=\varepsilon_{\infty}+\frac{\Delta \varepsilon}{\left[1+\left(i \omega \tau_{H N}\right)^{\alpha_{H N}}\right]^{\beta_{H N}}}+\frac{\sigma_{0}}{i \omega \varepsilon_{0}}
$$

where $\varepsilon^{\prime}$ and $\varepsilon^{\prime \prime}$ are respectively real and imaginary part of the complex dielectric function $\varepsilon^{*}, \varepsilon_{\infty}$ the dielectric constant in the high-frequency limit, $\Delta \varepsilon$ is the dielectric strength, $\tau_{\mathrm{HN}}$ relaxation time of $\mathrm{HN}$ function, $\sigma_{0}$ the de-conductivity, $\varepsilon_{0}$ the permittivity of vacuum. Exponents $\alpha_{\mathrm{HN}}$ and $\beta_{\mathrm{HN}}$ depict symmetric and asymmetric broadenings of the dielectric loss curve (with $0 \leq \alpha_{\mathrm{HN}} ; \beta_{\mathrm{HN}} \leq 1$ ). A specific case of $\alpha_{\mathrm{HN}}=\beta_{\mathrm{HN}}=1$ corresponds to a so-called Debye process which display a characteristic full width at half-maximum of 1.14 decades.

Supercooled liquid dynamics of Quinidine (i.e. dependence of structural $\alpha$-relaxation times) were described by the Vogel-Fulcher-Tammann (VTF) equation:

$$
\tau_{\alpha}(T)=\tau_{0} \exp \left(\frac{A}{T-T_{0}}\right)(\text { Eq. S1) }
$$

where $\tau_{0}, \mathrm{~T}_{0}$ and A are fitting parameters. We must specify that the VTF expression is sometimes adapted by substituting the parameter $\mathrm{A}$ by the expression $\mathrm{DT}_{0}$, where $\mathrm{D}$ is the fragility strength parameter. $\mathrm{T}_{0}$ is the so-called Vogel temperature regularly assigned as a hypothetical 
thermodynamic temperature at which cooperative molecular mobility is regarded to be close to zero.

Glassy dynamics of Quinidine (i.e. dependence of the secondary $\gamma$-relaxation times) were determined with the following Arrhenius equation:

$$
\tau(T)=\tau_{0} \exp \left(\frac{E_{a}}{R T}\right)(\text { Eq. S2) }
$$

where $\mathrm{R}$ is the universal gas constant, $\tau_{0}$ a pre-exponential factor and $\mathrm{E}_{\mathrm{a}}$ the activation energy.

Estimation of $\alpha$-relaxation times below $\mathrm{T}_{\mathrm{g}}$, was performed with a fitting process including a sum of two relaxation peaks. At $\mathrm{T}_{\mathrm{g}}$, the dielectric loss spectra corresponding to the $\alpha$-process was selected and fitted with a one-sided Fourier transform of a KWW function. The latter was shifted horizontally to temperatures below $\mathrm{T}_{\mathrm{g}}$ in order to match the high-frequency sided with the lowfrequency side of the spectra collected below $\mathrm{T}_{\mathrm{g}}$. To avoid any incorrect interpretations, a highfrequency contribution to fit the excess wing in the flank of the $\alpha$-loss peak through a Cole-Cole (CC) function was added.

\section{Molecular dipole moment decomposition:}

Within Bader's atoms-in-molecules theory (QTAIM) that partitions real space into non-overlapping atomic basins, $\Omega_{i}$, separated by "zero-flux" (of the electron density gradient) surfaces, the molecular dipole moment is exactly decomposed following:

$$
\vec{\mu}^{m o l}=\underbrace{\sum_{i}\left(q_{i} \vec{R}_{i}\right)}_{\sum_{i} \vec{\mu}_{i}^{\text {inter }}=\vec{\mu}^{\text {inter }}}+\underbrace{\sum_{i}\left(-\int_{\Omega_{i}} \rho(\vec{r})\left(\vec{r}-\vec{R}_{i}\right) d^{3} r\right)}_{\sum_{i} \vec{\mu}_{i}^{\text {pol }}=\vec{\mu}^{\text {pol }}},
$$

where $q_{i}, \vec{R}_{i}, \rho(\vec{r})$ denote atomic charge, nucleus location and electron density, respectively. The interatomic term arises from electron transfers between atomic basins, identical to a pure point charges description, while the polarization term represents the internal reorganization of the electron density inside each atomic basin upon bond formation. 
For a neutral molecule composed of fragments $\mathrm{A}$ and $\mathrm{B}$, the charge transfer amount is defined by:

$$
q=\left|\sum_{\text {atoms }_{i} \in A} q_{i}\right|=\left|\sum_{\text {atoms }_{j} \in B} q_{j}\right| .
$$

